\title{
Is There a Link between Suckling and Manipulation Behavior during Rearing in Pigs?
}

\author{
Friederike K. Warns ${ }^{1, *}$, Mehmet Gültas ${ }^{2,3,4, *(\mathbb{D})}$, Astrid L. van Asten ${ }^{5}$, Tobias Scholz $^{6}$ and Martina Gerken ${ }^{1}$ \\ 1 Department of Animal Science, Ecology of Livestock Production, University of Goettingen, \\ Albrecht-Thaer-Weg 3, 37075 Goettingen, Germany; mgerken@gwdg.de \\ 2 Department of Animal Science, Breeding Informatics Group, University of Goettingen, \\ Margarethe-von-Wrangell-Weg 7, 37075 Goettingen, Germany \\ 3 Center for Integrated Breeding Research (CiBreed), Albrecht-Thaer-Weg 3, Georg-August University, \\ 37075 Göttingen, Germany \\ 4 Faculty of Agriculture, South Westphalia University of Applied Sciences, Lübecker Ring 2, \\ 59494 Soest, Germany \\ 5 Department of Animal Production, Agricultural Chamber of North Rhine-Westphalia, Haus Duesse, \\ 59505 Bad Sassendorf, Germany; astrid.vanasten@1wk.nrw.de \\ 6 Agricultural Test Center VBZL Haus Duesse, Agricultural Chamber of North Rhine-Westphalia, Haus Duesse, \\ 59505 Bad Sassendorf, Germany; tobias.scholz@lwk.nrw.de \\ * Correspondence: friederike.warns@agr.uni-goettingen.de (F.K.W.); gueltas@cs.uni-goettingen.de (M.G.)
}

check for updates

Citation: Warns, F.K.; Gültas, M.; van Asten, A.L.; Scholz, T.; Gerken, M. Is There a Link between Suckling and Manipulation Behavior during Rearing in Pigs? Animals 2021, 11, 1175. https://doi.org/10.3390/ ani11041175

Academic Editor: Michaela Fels

Received: 1 April 2021

Accepted: 16 April 2021

Published: 20 April 2021

Publisher's Note: MDPI stays neutral with regard to jurisdictional claims in published maps and institutional affiliations.

Copyright: (c) 2021 by the authors. Licensee MDPI, Basel, Switzerland. This article is an open access article distributed under the terms and conditions of the Creative Commons Attribution (CC BY) license (https:// creativecommons.org/licenses/by/ $4.0 /)$.
Simple Summary: Tail biting, a well-known problem in modern pig production, reduces pigs' welfare and causes economic losses. It is influenced by several external and internal factors, such as housing condition, management, genetics, and age of the animals. Within the internal factors, the individual predisposition to tail biting is difficult to identify. In our study, we analyzed the manipulation behaviors of weaner pigs and their relationship with agonistic behaviors of the piglets during suckling to identify groups of piglets which showed similar suckling and rearing behaviors. In our experiment tail biting increased at the middle and end of rearing. Most animals were observed as both biters and victims of tail biting. During our observations, we found indications that tailbiting pigs showed mainly submissive behavior in teat disputes. These pigs might compensate their submissiveness by biting tails to chase other pigs from resources with restricted access, such as feed or enrichment material. Further research should consider more aspects of a pigs' personality suitable for early identification of pigs predisposed for later tail biting. This early identification would allow intervention measures to be taken earlier, thereby reducing tail biting and its consequences.

Abstract: Inadequate possibilities to perform oral manipulation behavior for pigs can lead to misdirection and thus tail biting. Our study aimed to analyze manipulation behaviors of weaner pigs with focus on tail biting and the relationship with agonistic characteristics of the piglets during suckling. We analyzed the individual manipulation behavior of 188 weaner pigs. General health condition and tail lesions were determined weekly. Correlations were estimated between weight at weaning and at the end of rearing period, frequency of manipulative rearing behaviors and Dominance and social tension index based on suckling behavior. Principal component and cluster analyses were performed to identify groups of piglets which showed similar suckling and rearing behaviors. Tail biting increased at the middle and end of rearing with switching roles of biters and victims. Tail lesions were correlated with received tail biting behavior but occurred with a delay of more than a week. The frequency of performed tail biting was correlated with dominance index $\left(\mathrm{r}_{\mathrm{s}}=-0.256\right.$, $p<0.01)$ and weaning weight $\left(\mathrm{r}_{\mathrm{s}}=-0.199, p<0.05\right)$. We assume that performed tail biting is more often observed in pigs who show mainly submissive behavior in teat disputes.

Keywords: pigs; tail biting; suckling behavior; dominance; rearing period; manipulation behavior 


\section{Introduction}

Pigs spend a significant amount of their time using oral manipulation to explore objects around them by sniffing, digging or chewing. This behavior can be redirected to other conspecifics, especially in barren housing systems [1,2], and can lead to various behaviors such as tail biting, ear biting or belly nosing [3]. Tail biting is one of the most important problems in modern pig production. Beside economic losses due to carcass trimming or full loss as consequence of spread infection, it causes stress, painful injuries and thereby reduced animal welfare in affected pigs [4-6].

Three different forms of tail biting are distinguished by the behavior of the performing pig: "two-stage tail biting", "sudden-forceful tail biting" and "obsessive tail biting" [7]. A tail biting outbreak may occur with usually one pig starting to bite and thereby encouraging further pen mates to join the behavior $[8,9]$. Within an outbreak, switching roles between performers and victims were observed [10,11]. Analysis of tail biting behavior is difficult because it occurs relatively rarely and seems unpredictable [12]. A wide range of influencing factors have been discussed by several authors (e.g., $[4,7,9,13-15])$. For example, refs. $[10,16,17]$ found lighter piglets to be more likely to bite pen mates and heavier piglets were bitten more frequently, whereas [18] found tail biters to be heavier than non-tail-biters. However, several authors failed to confirm significant correlations between a pigs' body weight and its frequency of performed tail biting $[11,19,20]$.

Several solutions were evaluated to prevent a tail biting outbreak such as environmental enrichment. Fewer wounded tails, more exploration behavior and improved animal welfare were found when pigs were offered organic objects and substrate which is ingestible, smellable, chewable, deformable, and destructible like roughage, wood shavings, or peas as enrichment material (e.g., [5,21-23]).

Another approach is to establish methods for early detection of occurrence of tail biting such as scoring of tail lesions or changes in behavioral traits. Tail biting outbreaks often seem to begin with first visible tail lesions two to three weeks after weaning, with the development of the percentage of injured pigs and the severity of the injuries depending on the design of the barn, including the enrichment material offered [9,24-29]. In many cases, these lesions are recognized with a delay of a few days after the frequency of tail biting behavior has already increased [30].

When analyzing the nosing behavior of pigs, Camerlink and Turner [31] found a low percentage of social nosing behavior that were followed by subsequent potentially injurious oro-nasal behavior. Within these behaviors, tail biting, ear biting and belly nosing were correlated with nosing of the corresponding body parts. In further studies positive correlations between tail biting and other manipulation behavior were found with biters spending more time to manipulate enrichment material than victims $[10,32]$ and also tended to bite more parts of the body [32,33] than non-tail-biters. In contrast, Larsen et al. [34] found no alteration of object manipulation prior to an outbreak but overall lower object manipulation rates in pens with tail damage. Furthermore, increased tail-inmouth behavior $[30,35]$ and activity level $[10,33,34]$, as well as an alteration of tail posture prior to a tail biting outbreak [36-41], are discussed as signs for early detection of tail biting.

Little is known whether predispositions for later tail biting can be already deduced from the piglets' behavior during the suckling period. Prunier et al. [12] reviewed several predisposing factors for biting experienced in early life as for example social stress due to fighting for teats or other resources. The authors pointed out an effect of this stress on aggressive biting during hierarchy formation but could not transfer this effect to non-aggressive forms such as tail biting [12]. Furthermore, Hoy et al. [11] did not find an association of certain teat positions during suckling with performed tail biting during rearing.

In the present study we followed individual agonistic behavior from suckling until the end of the rearing period at the age of 70 days. Analyses of suckling behavior is described in detail in Warns [42]. Here, we report on manipulation behavior of the weaned pigs with a special focus on performers and victims of tail biting across the rearing period of six 
weeks. Evaluation of general health condition and possible tail lesions of the pigs further provided additional information to the behavioral analysis. In a further step a possible relationship between agonistic behavior shown by a piglet during the suckling bouts and its manipulation behavior during rearing was analyzed. From these results, we wanted to deduce whether agonistic behavior shown during the competitive suckling situation could be used to predict tail biting during the later rearing phase.

\section{Materials and Methods}

The study was carried out at the Agricultural Test Center VBZL Haus Duesse of the Agricultural Chamber of North Rhine-Westphalia in Bad Sassendorf, Germany, between August 2017 and March 2018. The animals were kept according to the German Animal Protection Law and the Animal Welfare Livestock Farming Regulation.

The experiment was carried out in two repetition groups with a time interval of five weeks between trials. During suckling, twelve litters were housed in conventional farrowing crates $\left(4.58 \mathrm{~m}^{2}\right.$ per crate) and included 174 piglets across both repetitions. The environmental temperature during suckling was automatically regulated by forced ventilation and set at $23{ }^{\circ} \mathrm{C}$. The piglets had free access to a piglet nest with a heated floor plate, which was completed by a heat lamp for the first $48 \mathrm{~h}$ after birth. Usually, the animals had artificial light between 07:00 $\mathrm{h}$ and 16:00 $\mathrm{h}$ in addition to daylight via windows. During the entire suckling period the piglets had free access to water by a drinking trough. No enrichment material was offered to the piglets during suckling. Agonistic suckling behaviors of the piglets were analyzed individually (further details see [42]). Out of these piglets, we chose 59 weaner pigs per repetition for analysis of rearing behavior with a total of 118 pigs ( 59 castrated males and 59 females; Table 1) across both repetitions. The non-tail docked pigs were crossbreds of a Piétrain sire and a Topigs2 20 or Topigs70 dam and had an average birth weight of $1.44 \pm 0.34 \mathrm{~kg}$. Pigs were weaned with an age of 28 days and an average weight of $7.80 \pm 1.44 \mathrm{~kg}$. From day 21 after birth the pigs had ad libitum access to a feeding trough with a commercial rearing feed (feed A; 13.6 MJ ME, 15.0\% CP, 1.40\% Lys, $0.20 \% \mathrm{Na}$ ).

Table 1. Number of pigs, mean weaning and rearing weight $\pm S D$, number of dams and sex ratio per rearing pen.

\begin{tabular}{ccccccc}
\hline Repetition & Pen & Weaned Pigs (N) & $\begin{array}{c}\text { Average Weaning } \\
\text { Weight (kg) }\end{array}$ & $\begin{array}{c}\text { Dams } \\
\text { (N) }\end{array}$ & $\begin{array}{c}\text { Sex Ratio } \\
\text { Male:Female }\end{array}$ & $\begin{array}{c}\text { Average Weight at the } \\
\text { End of Rearing (kg) }\end{array}$ \\
\hline 1 & 1 & 9 & $6.12 \pm 0.21$ & 4 & $4: 5$ & $19.79 \pm 2.43$ \\
& 2 & 10 & $6.74 \pm 0.26$ & 5 & $6: 4$ & $22.03 \pm 2.62$ \\
& 3 & 10 & $7.30 \pm 0.22$ & 5 & $5: 5$ & $20.97 \pm 2.50$ \\
& 4 & 10 & $7.80 \pm 0.17$ & 5 & $5: 5$ & $23.06 \pm 2.34$ \\
2 & 5 & 10 & $8.53 \pm 0.23$ & 5 & $5: 5$ & $25.34 \pm 2.63$ \\
& 10 & $9.38 \pm 0.31$ & 4 & $5: 5$ & $25.72 \pm 2.55$ \\
& 1 & 10 & $6.60 \pm 0.23$ & 5 & $4: 5$ & $21.20 \pm 3.13$ \\
& 2 & 10 & $7.33 \pm 0.28$ & 5 & $5: 5$ & $21.23 \pm 3.46$ \\
& 3 & 10 & $7.96 \pm 0.15$ & 5 & $5: 5$ & $22.53 \pm 2.86$ \\
& 4 & 10 & $8.40 \pm 0.20$ & 5 & $5: 5$ & $22.51 \pm 2.01$ \\
& 5 & 10 & $9.08 \pm 0.27$ & 5 & $5: 5$ & $23.66 \pm 3.00$ \\
& 6 & & & & $5: 5$ & $25.10 \pm 1.84$ \\
\hline
\end{tabular}

From all pigs who reached a weaning weight of $6 \mathrm{~kg}$ or more, 59 pigs per repetition were chosen and distributed to rearing pens with nine (one pen) to ten pigs (five pens) each. By composing the groups, we considered similar weaning weight, a maximum of three pigs from the same litter and a balanced sex ratio in each group (Table 1).

The conventional rearing pens for ten pigs were sized $2.1 \times 1.7 \mathrm{~m}\left(0.36 \mathrm{~m}^{2}\right.$ per pig) with a fully slatted floor and no bedding material. In two pens, the available space was smaller because of a ventilation shaft, so only nine pigs were housed in these pens $\left(0.37 \mathrm{~m}^{2}\right.$ per pig). The environmental temperature was automatically regulated by forced ventilation and set on $29^{\circ} \mathrm{C}$ at the beginning of rearing (day 29 of life). During the subsequent weeks, 
it was decreased stepwise until $24.5^{\circ} \mathrm{C}$ at the end of rearing (day 70 of life). Usually, the animals had full artificial light (intensity: 90 lux in average) between 07:00 $\mathrm{h}$ and 16:00 $\mathrm{h}$ additionally to daylight. At the end of the rearing period, the pigs had an average weight of $22.80 \pm 3.10 \mathrm{~kg}$.

During the rearing period, the animals had ad libitum access to feed in a feeding trough and fresh water by drinking nipples. Feeding took place automatically by a dry feeding system. Until day 49 of life, the pigs received rearing feed A which they were used to from the last week of the suckling period. From day 50 of life, the diet was gradually changed during the next four days to rearing feed B (13.4 MJ ME, 16.0\% CP, 1.31\% Lys, $0.20 \% \mathrm{Na}$ ) which was fed until the end of the rearing period. The animal to feeding place ratio was $3: 1$ in pens with nine pigs $\left(3.31 \mathrm{~m}^{2}\right)$ and $3.3: 1$ in pens with ten pigs $\left(3.56 \mathrm{~m}^{2}\right)$. In each pen there was a metal chain with a plastic piece and a cotton rope provided as enrichment material. Twice a day, about $200 \mathrm{~g}$ of pellet mix of alfalfa, hay, corncob, and straw pellets were added to a pig bowl in each pen for oral manipulation. If tail biting occurred, pigs were offered a jute sack attached to the wall of the affected pen as additional enrichment material.

The individual body weight was measured weekly outside of the pens on a commercial stationary digital pig weight scale to the nearest $100 \mathrm{~g}$ (piglet scale, Meier-Brakenberg).

Possible tail lesions of each individual pig were scored weekly by the same observer during weighing. The individual general health condition was scored weekly in the pen using a scoring scheme modified from the Welfare Quality Assessment Protocol ${ }^{\circledR}$ for Pigs (Ref. [43], Table 2) and included lameness, injury of the ears, the carpal joints, and the body sides.

Table 2. Scoring scheme of the general health condition (modified after [43]).

\begin{tabular}{cccc}
\hline Parameter & Score $\mathbf{0}$ & Score 1 & Score 2 \\
\hline Lameness & No lameness & Lameness & - \\
Injury of the body sides & No injury & One side injured & Both sides injured \\
Injury of the carpal joints & No injury & One side injured & Both sides injured \\
Injury of the ears & No injury & One side injured & Both sides injured \\
\hline
\end{tabular}

The scoring scheme for possible tail lesions (modified from FLI [44]; Table 3) included tail length, hair coat, cleanliness, skin perforation, blood, and necrosis.

Table 3. Scoring scheme for possible tail lesions (German pig scoring system (Deutscher Schweine Bonitur Schlüssel) of FLI [44], modified).

\begin{tabular}{cccc}
\hline Parameter & Score $\mathbf{0}$ & Score 1 & Score 2 \\
\hline Tail length & Original length & Partial loss & Total loss (stump max. 1 cm) \\
Hair coat & Covered & Not covered & - \\
Cleanliness & Clean & Dirty & - \\
Skin perforation & No visible perforation & Superficial punctual perforation & Deeper skin perforation \\
Blood & No blood & Fresh blood & - \\
Necrosis & No necrosis & Necrosis, tissue change & - \\
\hline
\end{tabular}

During the entire suckling period each pen was equipped with a stationary HD camera (Dallmeier DF4820HD-DN/IR, 720 p, 6 fps) and the pigs were filmed continuously over the entire suckling phase of four weeks. The behavior of the pigs during 30 suckling bouts was analyzed for each pig individually as described in Warns [42]. In brief, based on the occurrence of agonistic traits during suckling, a dominance index [45] and social tension index [46] was calculated for each piglet individually. The dominance index was calculated by dividing the difference between the number of winds and defeats of social interactions by the sum of wins and defeats of social interactions [45]. For the social tension index, the difference between the sum of all the aggressive actions an animal has performed and the 
sum of all the aggressive actions an animal has received was calculated [46]. A positive dominance index of a piglet was the result of a high rate of success in teat disputes, whereas a high positive social tension index expressed a high willingness to initiate a teat dispute to gain access to a teat.

Similarly, during rearing, each rearing pen was equipped with a stationary HD camera (Dallmeier DF4820HD-DN/IR, 720 p, $6 \mathrm{fps}$ ) and the pigs were filmed continuously over the entire rearing phase of six weeks. For evaluation of the individual behavior of the pigs, each animal was marked with individual geometric symbols on its back using a commercial animal marking spray (Raidex ${ }^{\circledR}$, Raidex $\mathrm{GmbH}$, Dettingen, Germany; red, green and blue). The first marking took place directly after transfer to the pens. The pigs were remarked every two to three days to avoid fading of the marks. To ensure individual identification of pigs, only videos taken during the time with artificial light (07:15 to 16:00 h) were chosen for further analysis. In each repetition, we analyzed two days per week resulting in a total of twelve observation days per pig and repetition (total of 24 observation days across both repetitions and pens).

Videos were analyzed by the same observer for each pig individually by using the software Mangold Interact $^{\circledR}$ (Version 14.3.9.0; Mangold International GmbH; Arnstorf, Germany). Six different behavioral patterns were coded, separated in animal directed and object directed behavior with three behavioral patterns each. The animal directed behavior included tail biting, ear biting and belly nosing. Tail and ear biting were recorded continuously within the first ten minutes of an hour from 08:00 $\mathrm{h}$ to 15:10 $\mathrm{h}$, including information of the performer and the victim. Additionally, the reaction of the victim of tail biting was noted. For belly nosing and the three object directed behavioral patterns (manipulation of the rope, the pellets in the pig bowl, and the jute sack) the frequency of occurrence was determined by instantaneous scan sampling with a 30 sec interval within the above-mentioned time frame. For these four behaviors, the performer was noted (Table 4).

The statistical procedures were performed with $\mathrm{R}$ (Version 1.2.1335, RStudio, PBC, Boston, MA, USA). For analyzing general health condition and tail lesions of the rearing period we converted the evaluated scores to binary data and compared the different scoring times by chi-square tests (function "chisq.test"; [47]). For illustration of the development of behavioral traits during the entire rearing period, manipulation behaviors were analyzed by calculating average frequencies per pig, hour, and observation day. For the continuously recorded tail and ear biting, we determined the individual frequency of the behavior within the first ten minutes of an hour, extrapolated this result to the total hour by multiplying the result by six (according to Zonderland et al. [30]) and then calculated the average frequency of behavior per pig, hour and observation day. The remaining manipulation behaviors (recorded by scan sampling) were calculated as percentage of sampling points per hour and day in which behaviors were shown. For this purpose, we divided the determined frequency of behaviors shown at sampling points by the amount of sampling points per observation day and calculated an average per pig, hour and observation day.

Further statistics were based on the frequencies of behavior per individual, which were summarized per behavior over the entire observation period. Analyses of variance were performed for performer and victim of tail and ear biting, belly nosing, rope, pellets and sack manipulation (function "aov"; [48]) with repetition, sow, sex and pen of the pigs as fixed effects. Parameter "sow" referred to the sow pigs were raised at, so it described not necessarily the genetic mother of the pig. Tukey post-hoc tests were performed by function "TukeyHSD" [49]. Additionally, Spearman rank correlation coefficients were calculated (function "cor.test"; [50]) with a confidence interval of 95\% between weaning weight, weight at the end of rearing period, frequency of analyzed manipulation behaviors during rearing and dominance index and social tension index of the suckling piglets. Scatterplots were used to visualize the relationships between dominance index, social tension index and performer and victim of tail biting by the function "ggplot" (package "ggplot2"; [51]). Principal component analysis was performed to evaluate relationships 
between the behavior traits of the suckling and rearing period (function "PCA" of package "FactoMineR"; [52]) and its result was visualized in a scree plot for percentages of explained variances (function "fviz_eig" of package "factoextra"; [53]) and bar plots (function "fviz_contrib" of package "factoextra"; [53]) for contribution of variables and individuals to Dimension 1-2. Cluster analysis was performed with function "eclust" (package "factoextra"; [53]) to determine two clusters. Results of cluster analysis were visualized with function "fviz_silhouette" (package "factoextra"; [53]). A high positive silhouette value $\left(\mathrm{S}_{\mathrm{i}}\right)$ represented the similarity of an individual with its own cluster and poor matching with other clusters. Finally, principal component analysis was performed within the two calculated clusters as described above. Level of significance was set at $p<0.05$. Values are means \pm SD if not otherwise stated.

Table 4. Ethogram of the behavioral traits of the pigs during rearing.

\begin{tabular}{|c|c|c|}
\hline Behavior & Description & Observation Method \\
\hline & Animal directed behavior & \\
\hline Tail biting & $\begin{array}{l}\text { Visible tail biting: biting on the tail of a pig ("victim") by another pig } \\
\text { ("performer"); visible tail-in-mouth behavior: light chewing on the tail of a } \\
\text { victim by a performer; new action counted after an interruption of at least } 3 \mathrm{~s} \\
\text { with no contact between the snout of the performer and victim's tail } \\
\rightarrow \text { Reaction of the victim: }\end{array}$ & $\begin{array}{l}\text { Continuously, } \\
\text { individual performer } \\
\text { and victim, reaction of } \\
\text { victim }\end{array}$ \\
\hline Tolerate & No visible reaction as a consequence of tail biting & \\
\hline Avoid & $\begin{array}{l}\text { Pulling in the tail, move away (slides forward while lying) or walking } \\
\text { away calmly }\end{array}$ & \\
\hline Jump up & $\begin{array}{l}\text { Sudden rising of the animal from a lying or resting position; sudden leap } \\
\text { forward when the animal was already standing }\end{array}$ & \\
\hline Blood & $\begin{array}{l}\text { Visible fresh blood on the victim's tail immediately after a bite; recorded only } \\
\text { on first appearance, not on follow-up bites on the same pig when fresh blood } \\
\text { was already visible }\end{array}$ & \\
\hline Ear biting & $\begin{array}{l}\text { Visible ear biting or chewing on the ear of a pig ("victim") by another pig } \\
\text { ("performer"); new action counted after an interruption of at least } 3 \mathrm{~s} \text { with no } \\
\text { contact between the snout of the performer and victim's ear }\end{array}$ & $\begin{array}{l}\text { Continuously, } \\
\text { individual performer } \\
\text { and victim }\end{array}$ \\
\hline Belly nosing & $\begin{array}{l}\text { Rhythmically raising and lowering the snout of a pig ("performer") with } \\
\text { pressure in the area of the abdominal cavity of another pig while it was lying; } \\
\text { at least } 2 \mathrm{~s} \text { of continuous movement }\end{array}$ & $\begin{array}{l}\text { Scan sampling, } \\
\text { performer }\end{array}$ \\
\hline $\begin{array}{l}\text { Cotton rope and metal } \\
\text { chain with plastic } \\
\text { piece ("rope") }\end{array}$ & $\begin{array}{l}\text { Object orientated behavior } \\
\text { Visible "take in the mouth", chewing, holding or pulling on one of the } \\
\text { components; targeted searching, sniffing or moving one of the components } \\
\text { with the snout; visible movement of the components }\end{array}$ & $\begin{array}{l}\text { Scan sampling, } \\
\text { performer }\end{array}$ \\
\hline $\begin{array}{l}\text { Piglet bowl with pellet } \\
\text { mix ("pellets") }\end{array}$ & $\begin{array}{l}\text { Lowered head in or over the piglet bowl; visible manipulation of the bowl, } \\
\text { rooting under the outer edge of the bowl, chewing of bowl components } \\
\text { or pellets }\end{array}$ & $\begin{array}{l}\text { Scan sampling, } \\
\text { performer }\end{array}$ \\
\hline Jute sack ("sack") & $\begin{array}{l}\text { Visible "take in the mouth", chewing, holding or pulling on the jute sack; } \\
\text { targeted searching, sniffing or moving the sack with the snout; visible } \\
\text { movement of the sack }\end{array}$ & $\begin{array}{l}\text { Scan sampling, } \\
\text { performer }\end{array}$ \\
\hline
\end{tabular}

\section{Results}

\subsection{Weight Gain and Results of Injury Evaluation}

Pigs were assigned to the pens according to their weaning weight. Average daily weight gain was similar in both repetitions with $0.362 \pm 0.064$ and $0.355 \pm 0.066 \mathrm{~kg}$ in repetitions 1 and 2, respectively.

Evaluation results of general health condition and tail lesions were analyzed as binary system to describe the presence or absence of an injury (Table 5). The proportion of pigs with injured body sides increased in both repetitions ( 0 to $71 \%$ and 37 to $59 \%$, respectively) whereas the proportion of pigs with injured carpal joints decreased until the end of rearing period in both repetitions (61\% to $5 \%$ and $49 \%$ to $10 \%$, respectively). In both repetitions a 
high variation within the proportion of pigs with injured ears ( $2 \%$ to $22 \%$ in repetition 1 and $3 \%$ to $80 \%$ in repetition 2 ) occurred whereas the frequency of lameness was very low.

Table 5. Scored parameters with proportion (\%) of pigs for each score.

\begin{tabular}{|c|c|c|c|c|c|c|c|c|c|c|c|c|}
\hline \multirow[t]{2}{*}{ Parameter } & \multicolumn{6}{|c|}{$\begin{array}{c}\text { Repetition } 1 \\
\text { Day of Life }\end{array}$} & \multicolumn{6}{|c|}{$\begin{array}{c}\text { Repetition } 2 \\
\text { Day of Life }\end{array}$} \\
\hline & 35 & 42 & 49 & 56 & 63 & 70 & 35 & 42 & 49 & 56 & 63 & 70 \\
\hline \multicolumn{13}{|c|}{ General health condition } \\
\hline Lameness & $2 \%{ }^{a}$ & $0 \% \mathrm{a}$ & $0 \%{ }^{a}$ & $0 \%{ }^{a}$ & $0 \%^{a}$ & $0 \%$ a & $0 \%$ a & $0 \%{ }^{a}$ & $0 \%{ }^{a}$ & $0 \%{ }^{a}$ & $0 \%$ a & $0 \%{ }^{\mathrm{a}}$ \\
\hline $\begin{array}{l}\text { Body side(s) } \\
\text { injured }\end{array}$ & $0 \%{ }^{\text {a }}$ & $0 \%{ }^{\text {a }}$ & $0 \%{ }^{\text {a }}$ & $24 \% \mathrm{~b}, \mathrm{c}$ & $56 \%$ d,e & $71 \%^{d}$ & $37 \%$ c,e & $0 \%$ a & $5 \%$ a & $15 \% \mathrm{~b}$ & $38 \%{ }^{c, e}$ & $59 \%$ d,e \\
\hline $\begin{array}{l}\text { Carpal joint(s) } \\
\text { injured }\end{array}$ & $61 \%^{a}$ & $34 \%{ }^{a}$ & $51 \%$ a,b & $39 \%$ a,b & $0 \%{ }^{c}$ & $5 \% c, d$ & $49 \%{ }^{a, b}$ & $31 \% \mathrm{~b}$ & $37 \%$ a,b & $61 \%{ }^{a}$ & $2 \% c, d$ & $10 \%{ }^{d}$ \\
\hline Ear(s) injured & $15 \%^{a, b}$ & $5 \%$ b,c & $12 \%$ & $2 \%^{c}$ & $19 \%$ a & $22 \%^{a}$ & $80 \%{ }^{d}$ & $20 \%{ }^{a}$ & $8 \%{ }^{a, b, c}$ & $20 \%{ }^{a}$ & $3 \% b, c$ & $17 \%^{\mathrm{a}}$ \\
\hline \multicolumn{13}{|c|}{ Tail lesions } \\
\hline Fresh blood & $2 \%^{a}$ & $0 \%{ }^{\text {a }}$ & $0 \%{ }^{\text {a }}$ & $8 \%$ b,c & $22 \%{ }^{c, d}$ & $36 \%^{d}$ & $0 \%{ }^{\mathrm{a}}$ & $0 \%{ }^{\mathrm{a}}$ & $0 \%{ }^{a}$ & $0 \%{ }^{\mathrm{a}}$ & $7 \%{ }^{b}$ & $15 \% \mathrm{~b}, \mathrm{c}$ \\
\hline $\begin{array}{c}\text { Not covered with } \\
\text { hair }\end{array}$ & $3 \%$ a & $0 \%{ }^{\text {a }}$ & $5 \%$ a & $22 \% \mathrm{~b}, \mathrm{c}$ & $41 \% c$ & $51 \%{ }^{c}$ & $0 \%$ a & $0 \%$ a & $0 \%{ }^{a}$ & $0 \%$ a & $10 \%$ b & $2 \%$ \\
\hline $\begin{array}{l}\text { Visible and deeper } \\
\text { skin perforation }\end{array}$ & $5 \%{ }^{\mathrm{a}, \mathrm{e}}$ & $3 \%^{a}$ & $10 \%{ }^{a, b}$ & $17 \%$ b,c,e & $47 \%$ d & $64 \%{ }^{d}$ & $5 \%$ a & $12 \%{ }^{a, b}$ & $0 \%{ }^{a}$ & $15 \%$ b,c,e & $25 \%{ }^{c}$ & $47 \%^{\mathrm{d}}$ \\
\hline Partial loss & $0 \%^{\mathrm{a}}$ & $0 \%{ }^{a}$ & $0 \%^{\mathrm{a}}$ & $12 \%$ b & $24 \%{ }^{b, c}$ & $29 \%{ }^{c}$ & $2 \%{ }^{a}$ & $0 \%{ }^{a}$ & $0 \%{ }^{a}$ & $0 \%{ }^{a}$ & $5 \%^{a}$ & $15 \% \mathrm{~b}, \mathrm{c}$ \\
\hline $\begin{array}{c}\text { Necrosis, tissue } \\
\text { change }\end{array}$ & $2 \%$ & $0 \%^{\text {a }}$ & $2 \%$ & $7 \% \mathrm{~b}, \mathrm{c}$ & $19 \%^{c}$ & $14 \%^{c}$ & $0 \%{ }^{a}$ & $0 \%{ }^{a}$ & $0 \%{ }^{a}$ & $0 \%{ }^{a}$ & $15 \%{ }^{c}$ & $2 \%^{a, b}$ \\
\hline Covered with dirt & $10 \%^{a}$ & $25 \%$ b,c & $27 \%$ b,c & $37 \% \mathrm{~b}, \mathrm{~d}$ & $44 \% \mathrm{~b}$ & $44 \% \mathrm{~b}$ & $31 \% \mathrm{~b}, \mathrm{c}, \mathrm{d}$ & $25 \% \mathrm{~b}, \mathrm{c}$ & $41 \%^{\mathrm{b}}$ & $19 \%$ a,c,d & $29 \%$ b,c & $14 \%{ }^{a, c}$ \\
\hline
\end{tabular}

$\mathrm{a}, \mathrm{b}, \mathrm{c}, \mathrm{d}, \mathrm{e}$ terms with different letters within one row differ significantly by repetition $(p<0.05)$; tested with absolute frequencies by chi-squared test.

Overall, the proportion of pigs with tail lesions increased in both repetitions until the end of the rearing period with considerable variations between repetitions. The proportion of pigs with tails with fresh blood ranged between 15-36\% and partial losses were noted in $15-29 \%$ of the animals at the end of the rearing period.

\subsection{Development of Manipulative Behaviour during the Rearing Period}

Analyses of variance showed a significant effect $(p<0.001)$ of repetition on frequency of received tail biting with a higher average in repetition $1(0.35 \pm 0.25$ received tail biting behaviors per hour and pig) than in repetition $2(0.20 \pm 0.14$ received tail biting behaviors per hour and pig). In addition, a significant effect $(p=0.008)$ of pen with a high average in pen 5 of repetition $1(0.61 \pm 0.37$ received tail biting behaviors per hour and pig) was found. The frequency of performed tail biting was not affected by repetition, sow, sex, and pen (Table 6).

Table 6. Average manipulation behavior during rearing, frequency ${ }^{1}$ or percentage ${ }^{2}$ per pig and hour.

\begin{tabular}{|c|c|c|c|c|c|c|c|c|}
\hline Parameter & $\begin{array}{l}\text { Performer Tail } \\
\text { Biting }(\mathrm{n} / \mathrm{h})\end{array}$ & $\begin{array}{l}\text { Victim Tail } \\
\text { Biting (n/h) }\end{array}$ & $\begin{array}{l}\text { Performer Ear } \\
\text { Biting }(\mathrm{n} / \mathrm{h})\end{array}$ & $\begin{array}{l}\text { Victim Ear } \\
\text { Biting (n/h) }\end{array}$ & $\begin{array}{c}\text { Belly } \\
\text { Nosing (\%) }\end{array}$ & Rope (\%) & Pellets (\%) & Sack (\%) \\
\hline Mean \pm SD & $0.27 \pm 0.48$ & $0.27 \pm 0.22$ & $0.13 \pm 0.11$ & $0.13 \pm 0.11$ & $0.30 \pm 0.31$ & $3.75 \pm 1.38$ & $3.41 \pm 1.45$ & $0.20 \pm 0.51$ \\
\hline \multicolumn{9}{|c|}{ Analysis of variance ( $p$ value) } \\
\hline Repetition & 0.083 & $<0.001$ & 0.026 & 0.020 & 0.005 & 0.122 & 0.383 & $<0.001$ \\
\hline Sow & 0.187 & 0.626 & 0.528 & 0.286 & $<0.001$ & 0.002 & 0.050 & $<0.001$ \\
\hline Sex & 0.381 & 0.928 & 0.575 & 0.056 & 0.278 & 0.034 & 0.472 & 0.658 \\
\hline Pen & 0.953 & 0.008 & 0.102 & 0.125 & 0.002 & 0.006 & 0.755 & $<0.001$ \\
\hline
\end{tabular}

${ }^{1}$ Continuous recording of individual frequency; ${ }^{2}$ Individual percentage of sampling points.

In the first repetition the average frequency of tail biting incidences per pig and observation day increased stepwise until a first peak at day 52 of life ( 0.52 tail biting behaviors per hour and pig) and reached its maximum at day 65 of life (1.37 tail biting behaviors per hour and pig; Figure 1a). Until the first peak the majority of tail biting incidents were tolerated by the victim. Subsequently, the proportion of responses "avoid" or "jump up" by the victim increased until the end of the rearing period. Tail biting behavior resulting in fresh bloody injuries was observed only rarely. In repetition 2 , the average frequency of tail biting behavior varied between observation days with a maximum of 
0.31 tail biting incidents per hour and pig on day 47 of life and mostly tolerating reactions of the victims.

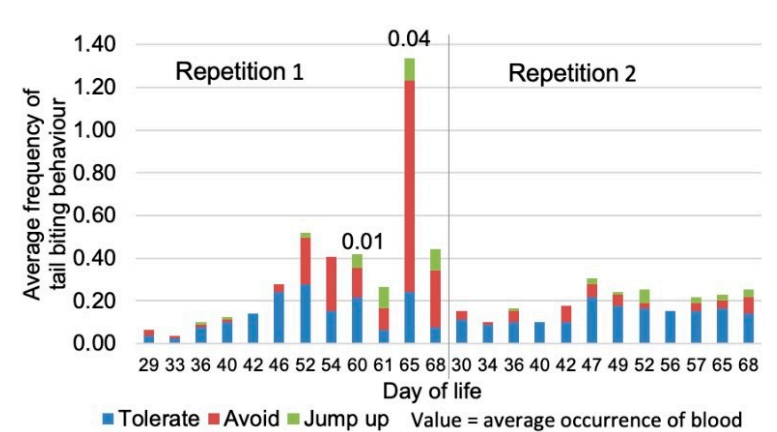

(a)

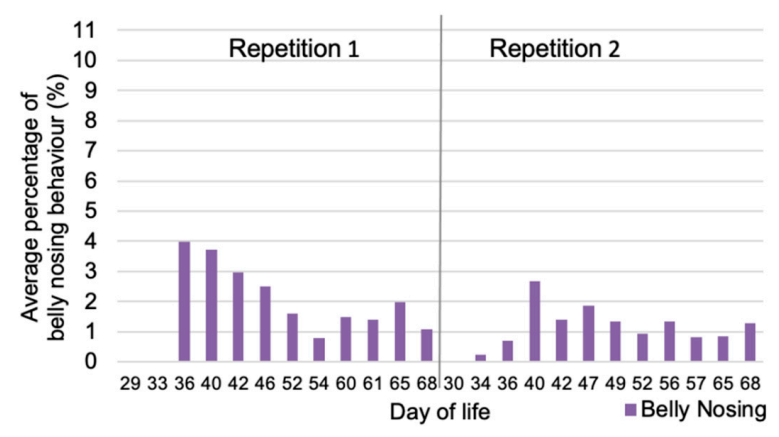

(c)

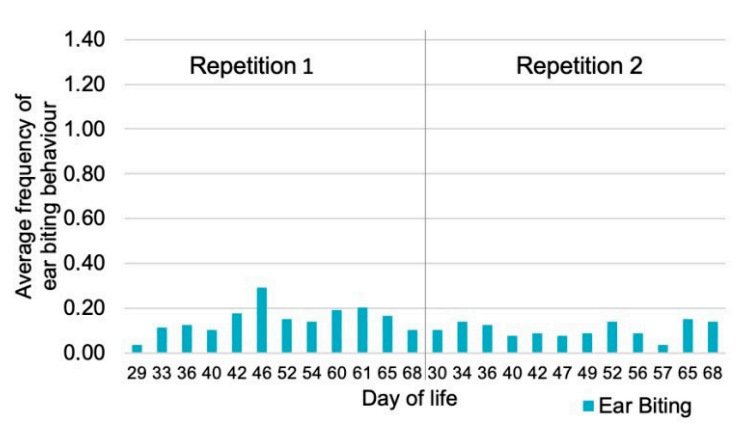

(b)

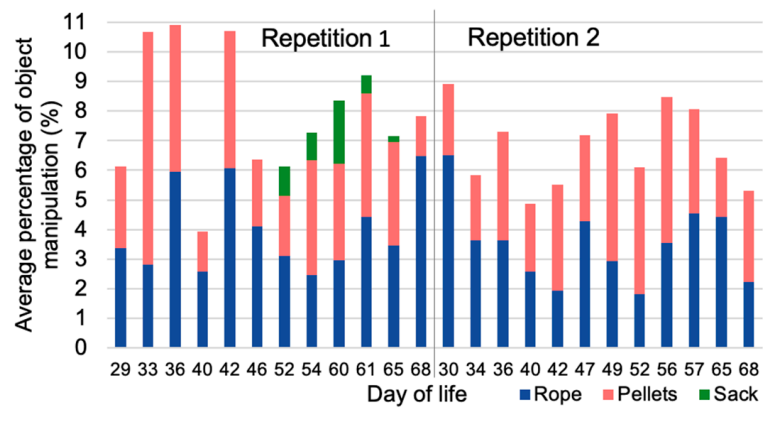

(d)

Figure 1. Average frequency and percentage of manipulation behavior per pig and hour by observation day of the rearing period by repetition (a) average frequency of tail biting with victims' reaction per pig; (b) average frequency of ear biting per pig; (c) belly nosing behavior per pig as percentage of sampling points; (d) object manipulation per pig as percentage of sampling points.

The average frequency of ear biting varied on a low level between observation days (Figure 1b). The average frequency of ear biting was significantly higher in repetition 1 $(0.15 \pm 0.12$ behaviors per hour and pig) than in repetition $2(0.10 \pm 0.10$ behaviors per hour and pig) but not influenced by further tested effects.

Among belly nosing behavior (Figure 1c) the highest average frequencies per sampling points was observed in both repetitions in the second week after weaning (days 36 and 40 of life), followed by varying but overall decreasing proportions until the end of the rearing period. Belly nosing was affected by repetition $(p=0.005)$ with a higher average in repetition $1(0.36 \pm 0.37 \%$ of sampling points) than in repetition $2(0.23 \pm 0.22 \%$ of sampling points), and was also affected by pen with a significantly $(p=0.002)$ high average in pen 2 of repetition $1(0.62 \pm 0.45 \%$ of sampling points), and by sow.

All object directed manipulation behaviors ("rope", "pellets" and "sack"; Figure 1d) were observed in varying frequencies and affected by sow. Rope manipulation behavior was also significantly affected by sex and pen with higher averages shown in male $(4.09 \pm 2.04 \%$ of sampling points) than in female pigs ( $3.40 \pm 1.78 \%$ of sampling points) and a significant low average in pen 6 of repetition 1 ( $2.46 \pm 0.57 \%$ of sampling points). Manipulation of the sack was only observed on days 52 to 65 of life in three pens of repetition 1 . The sack was only offered in three pens of repetition 1 with severe tail biting, which strongly biased the effects of repetition, sow and pen.

\subsection{Dominance and Social Tension Index from the Suckling Period}

For allocation to the rearing pens at weaning, littermates were distributed across different pens. The resulting mean indices per rearing pen (Table 7) showed high standard 
deviations. Highest mean dominance indices were found in pens 5 and 6 of repetition 1 whereas the mean social tension index was lowest in pen 2 of repetition 1 (Table 7).

Table 7. Means $\pm S D$ and range of Dominance Index and Social Tension Index ${ }^{1}$ per rearing pen and repetition.

\begin{tabular}{|c|c|c|c|c|c|c|c|}
\hline \multirow[b]{2}{*}{ Repetition } & \multirow[b]{2}{*}{ Pen } & \multicolumn{3}{|c|}{ Dominance Index } & \multicolumn{3}{|c|}{ Social Tension Index } \\
\hline & & Mean \pm SD & $\min$ & $\max$ & Mean \pm SD & $\min$ & $\max$ \\
\hline 1 & 1 & $0.08 \pm 0.36$ & -0.58 & 0.49 & $-3.33 \pm 13.24$ & -25 & 22 \\
\hline 1 & 2 & $0.05 \pm 0.24$ & -0.31 & 0.45 & $-6.10 \pm 10.56$ & -27 & 5 \\
\hline 1 & 3 & $-0.03 \pm 0.33$ & -0.60 & 0.35 & $-2.20 \pm 9.60$ & -15 & 13 \\
\hline 1 & 4 & $0.09 \pm 0.29$ & -0.56 & 0.48 & $-3.60 \pm 9.85$ & -19 & 11 \\
\hline 1 & 5 & $0.20 \pm 0.33$ & -0.12 & 0.86 & $1.50 \pm 6.98$ & -8 & 18 \\
\hline 1 & 6 & $0.22 \pm 0.21$ & -0.11 & 0.58 & $0.90 \pm 8.23$ & -9 & 18 \\
\hline 2 & 1 & $-0.08 \pm 0.38$ & -0.51 & 0.64 & $-0.11 \pm 7.75$ & -12 & 11 \\
\hline 2 & 2 & $-0.07 \pm 0.32$ & -0.67 & 0.37 & $-4.10 \pm 6.89$ & -18 & 3 \\
\hline 2 & 3 & $0.01 \pm 0.18$ & -0.33 & 0.29 & $3.10 \pm 6.76$ & -8 & 13 \\
\hline 2 & 4 & $0.06 \pm 0.31$ & -0.47 & 0.55 & $-1.70 \pm 8.30$ & -12 & 14 \\
\hline 2 & 5 & $0.05 \pm 0.35$ & -0.60 & 0.67 & $0.80 \pm 7.35$ & -6 & 18 \\
\hline 2 & 6 & $0.03 \pm 0.30$ & -0.39 & 0.63 & $-4.60 \pm 8.63$ & -17 & 8 \\
\hline
\end{tabular}

${ }^{1}$ Based on individual agonistic traits during the suckling period; for details see Warns [42].

\subsection{Correlations between Traits}

Most correlations were of low magnitude (Table 8). A high correlation was found between body weights at the age of four and ten weeks. Among traits recorded during suckling, dominance index was closely negatively correlated with social tension index $\left(r_{s}=-0.466, p<0.001\right.$; Table 8). However, the relationship between dominance index and social tension index with the manipulative behavior during rearing was low. Only between dominance index and tail biting performer a moderate negative correlation of -0.256 $(p=0.005$; Figure 2$)$ was found. Traits of tail lesions were consistently correlated with tail biting receiver $\left(r_{s}=0.265\right.$ to 0.379$)$. The correlation with "sack" is biased, because only in the case of tail biting occurrence was a sack provided.

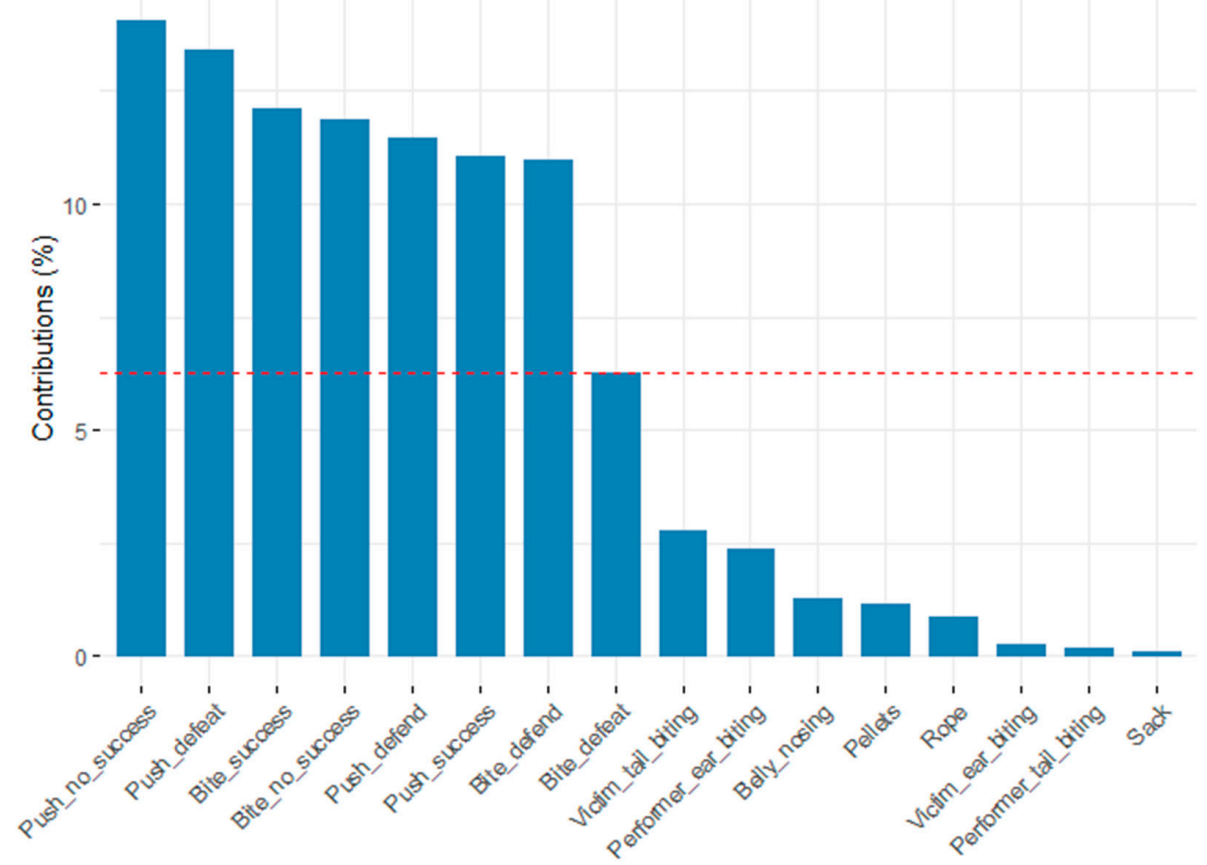

Figure 2. Contribution of variables to principal component factors 1 and 2 for all pigs; dashed line $=$ expected value if contribution were uniform. 


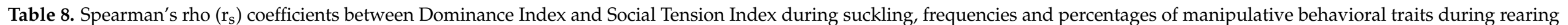
and general health condition and tail lesions at the end of the rearing period.

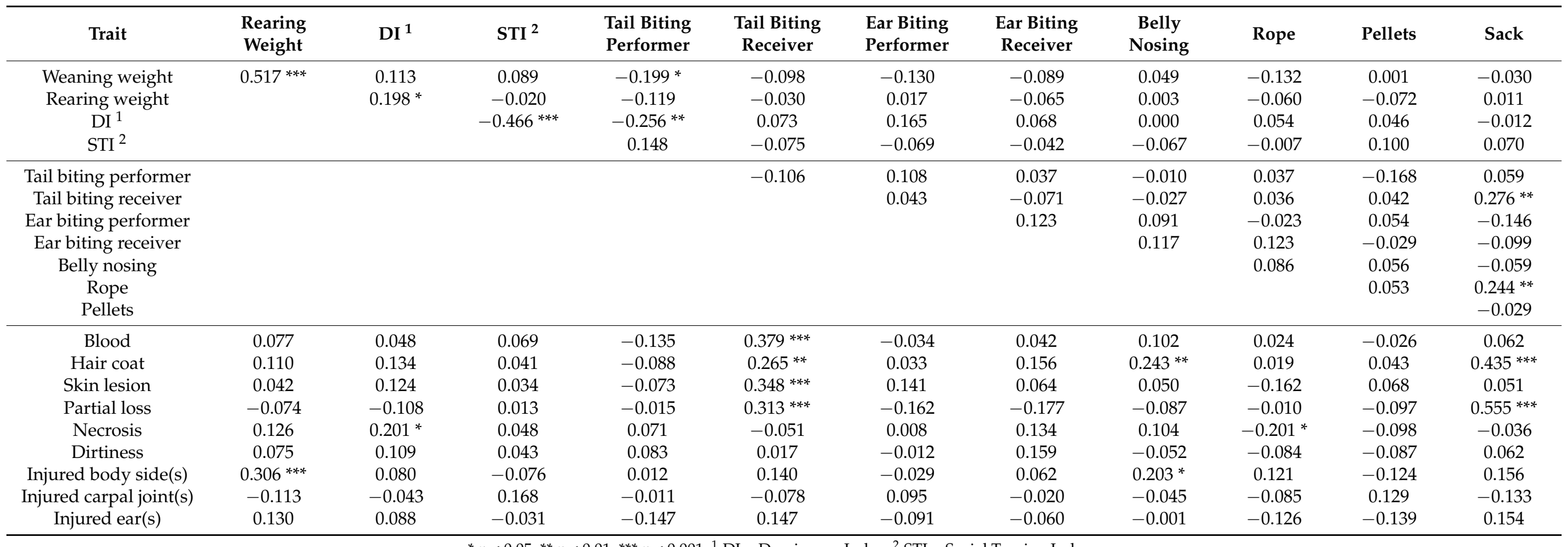

${ }^{*} p<0.05,{ }^{* *} p<0.01,{ }^{* * *} p<0.001,{ }^{1}$ DI $=$ Dominance Index, ${ }^{2}$ STI = Social Tension Index. 


\subsection{Principal Components and Clusters}

Table 9 lists the outcomes of the principal component analysis (PCA) performed on the frequency of agonistic suckling behaviors and manipulative rearing behaviors. Five main factors were identified with Eigenvectors $>1$, which together explained $54.953 \%$ of the variation between pigs. Principal component factors 1 and 2 explained $30.572 \%$ of the variation between the pigs (Table 9). The highest contributions to these principal components were shown by seven of the eight suckling behaviors (Figure 2).

Table 9. Outcomes of the Principal Component Analysis of suckling and rearing behaviors with Eigen value $>1$.

\begin{tabular}{|c|c|c|c|c|c|}
\hline Behavior & PC 1 & PC 2 & PC 3 & PC 4 & PC 5 \\
\hline Eigen value & 3.117 & 1.774 & 1.447 & 1.326 & 1.129 \\
\hline Variance explained (\%) & 19.484 & 11.088 & 9.041 & 8.286 & 7.054 \\
\hline Variance explained (\%, cumulative) & 19.484 & 30.572 & 39.613 & 47.899 & 54.953 \\
\hline \multicolumn{6}{|l|}{ Suckling behavior 1} \\
\hline Push-success & 0.638 & -0.365 & -0.267 & -0.042 & 0.194 \\
\hline Push-no success & 0.654 & -0.509 & 0.042 & 0.020 & 0.006 \\
\hline Bite-success & 0.591 & 0.494 & 0.157 & -0.147 & -0.266 \\
\hline Bite-no success & 0.565 & 0.510 & 0.067 & -0.072 & -0.282 \\
\hline Push-defeat & 0.716 & -0.378 & -0.076 & -0.033 & -0.010 \\
\hline Bite-defeat & 0.431 & 0.348 & 0.056 & -0.008 & 0.380 \\
\hline Push-defend & 0.733 & -0.149 & -0.066 & 0.017 & 0.062 \\
\hline Bite-defend & 0.528 & 0.507 & -0.083 & 0.228 & 0.043 \\
\hline \multicolumn{6}{|l|}{ Rearing behavior } \\
\hline Belly nosing & 0.168 & -0.185 & 0.586 & -0.077 & 0.056 \\
\hline Rope & 0.065 & -0.195 & 0.493 & 0.586 & 0.143 \\
\hline Pellets & -0.175 & -0.158 & 0.000 & -0.161 & 0.352 \\
\hline Sack & -0.043 & 0.039 & -0.187 & 0.750 & 0.106 \\
\hline Tail biting performer & -0.061 & 0.059 & 0.394 & 0.377 & -0.364 \\
\hline Tail biting receiver & 0.035 & 0.366 & -0.264 & 0.258 & 0.497 \\
\hline Ear biting performer & -0.135 & 0.313 & 0.277 & -0.291 & 0.423 \\
\hline Ear biting receiver & 0.106 & -0.008 & 0.632 & -0.110 & 0.272 \\
\hline
\end{tabular}

PC = Principal Component factor; highest loadings per behavior are in bold type; ${ }^{1}$ For detailed explanation of traits see Warns [42].

We performed a cluster analysis to identify similar groups of pigs based on their observed behaviors during both suckling and rearing. However, the visualization of the results in a silhouette plot (Figure 3) showed an overall low similarity of the pigs with their cluster (average $S_{i}=0.27$ ).

Within cluster 1, five main factors were identified with an Eigenvalue $>1$, explaining $53.84 \%$ of the variation between pigs (Figure 4 ). The total contribution of variables to principal components 1 and 2 (Figure 5) showed highest loadings for five suckling behaviors, "victim of tail biting" and "performer of ear biting".

Cluster 2 identified five main factors with an Eigenvalue $>1$, explaining $58.89 \%$ of the variation between pigs (Figure 6). Within principal components 1 and 2, the contribution of variables (Figure 7) showed highest loadings for the eight suckling behaviors. 


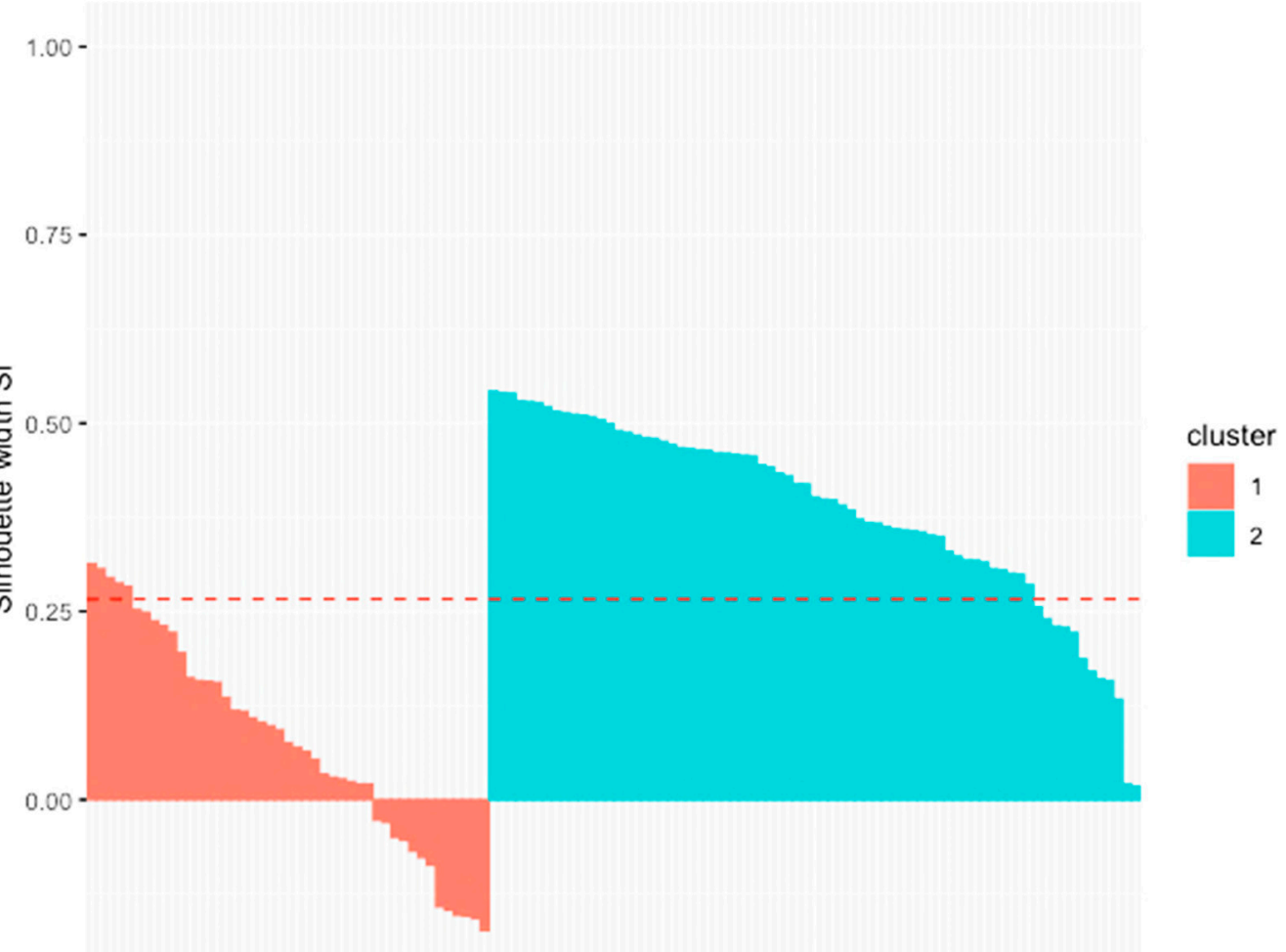

Figure 3. Cluster silhouette plot for categorization of piglets in two clusters based on their suckling and rearing behavior; dashed line $=$ average $S_{i}$.

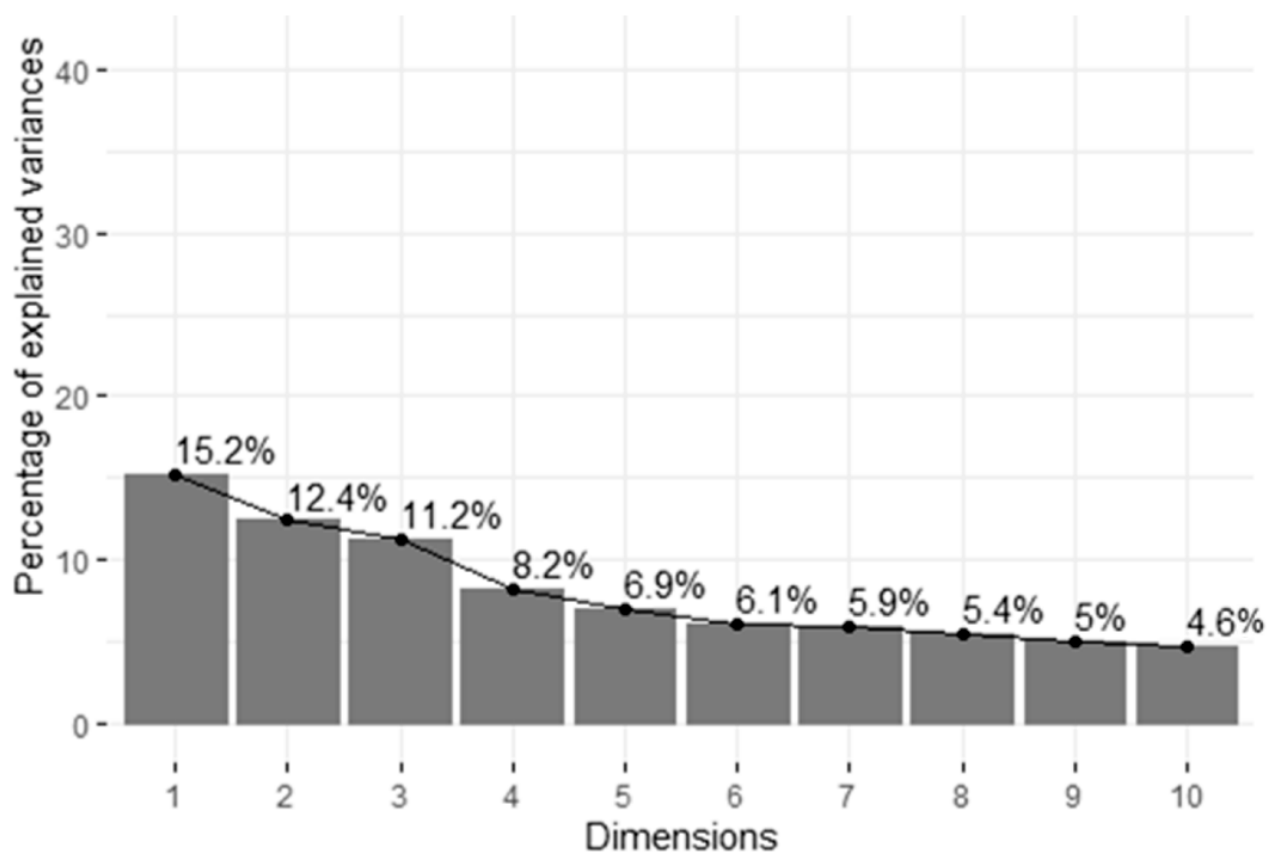

Figure 4. Scree plot with percentage of explained variances per principal component factors (dimensions) of cluster 1. 


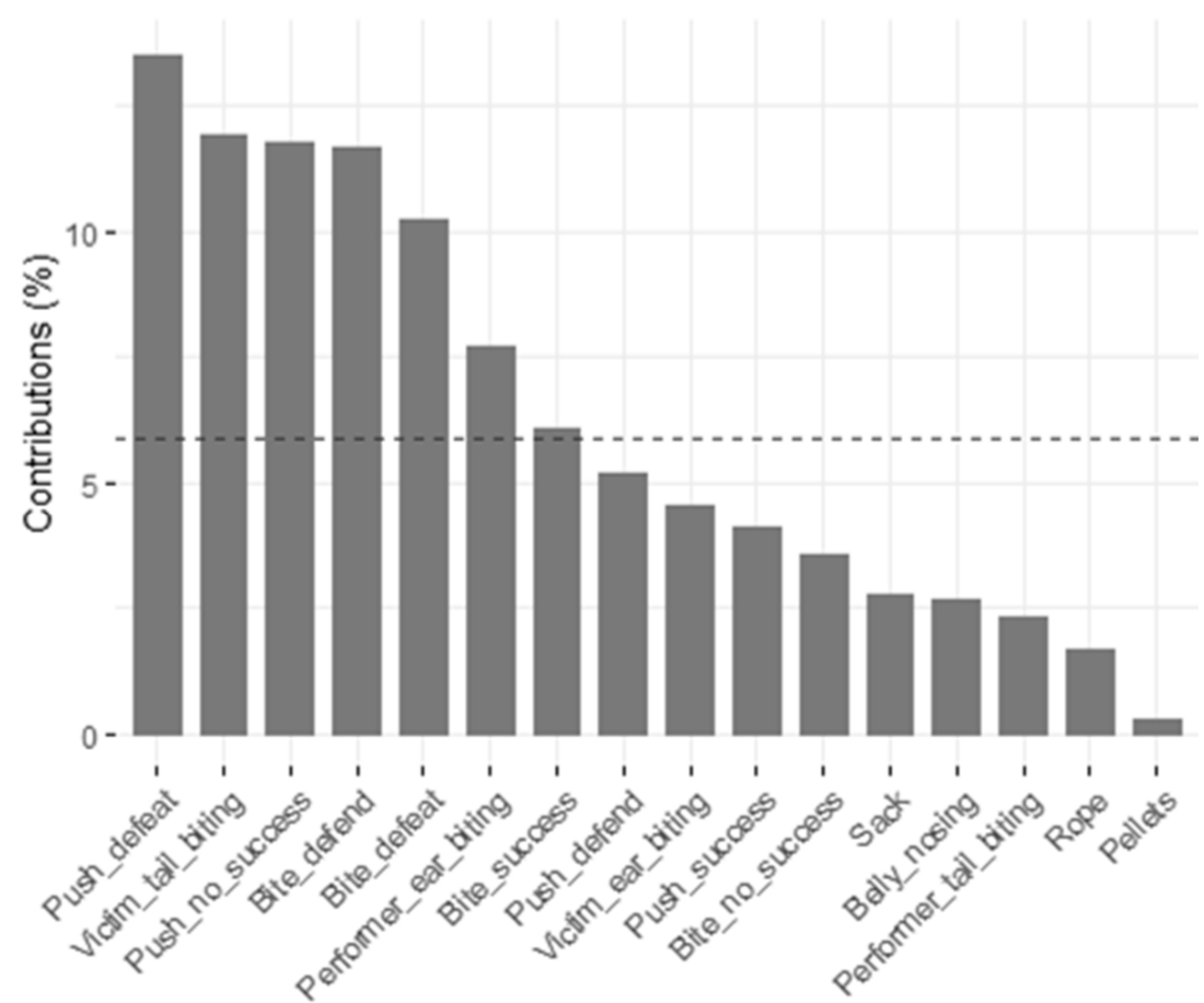

Figure 5. Contribution of variables to principal component factors 1 and 2 for cluster 1; dashed line $=$ expected value if contribution were uniform.

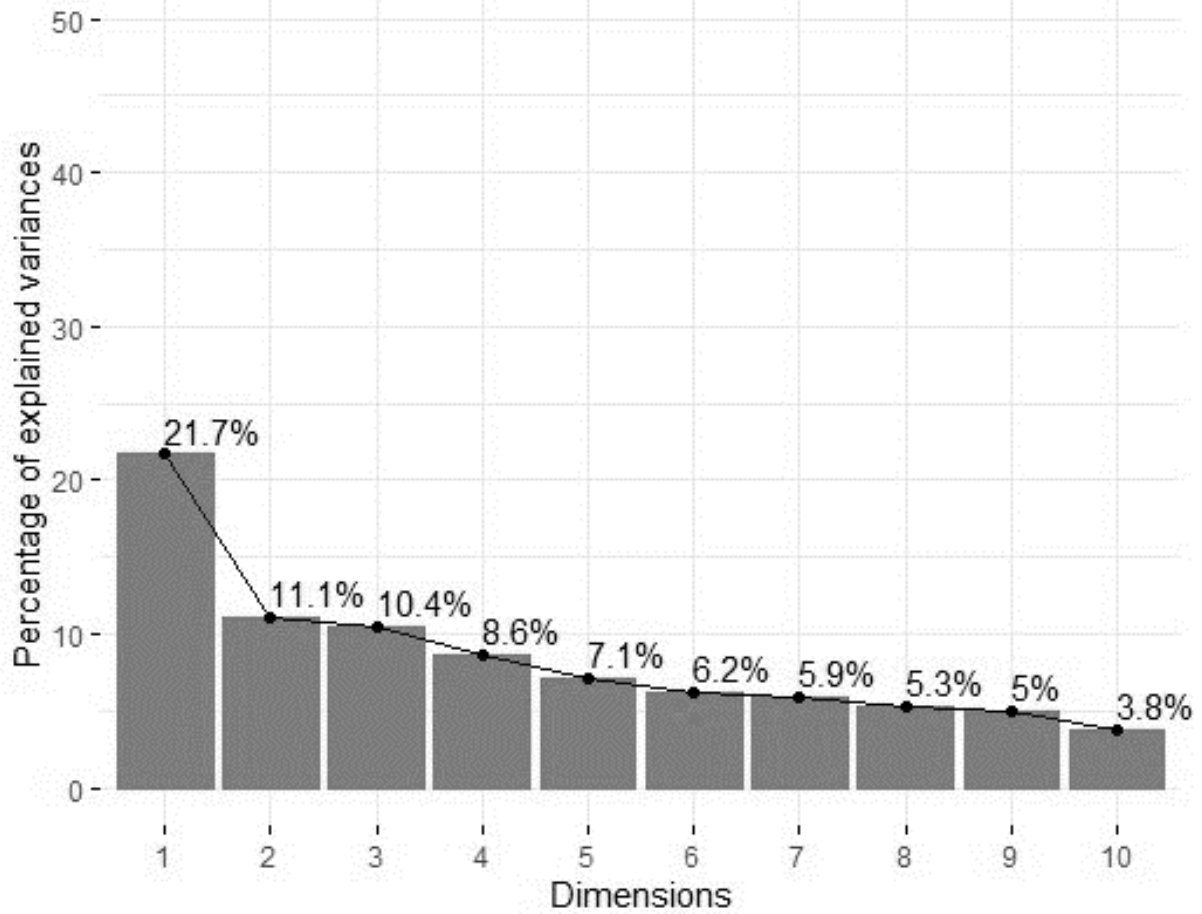

Figure 6. Scree plot with percentage of explained variances per principal component factors (dimensions) of cluster 2 . 


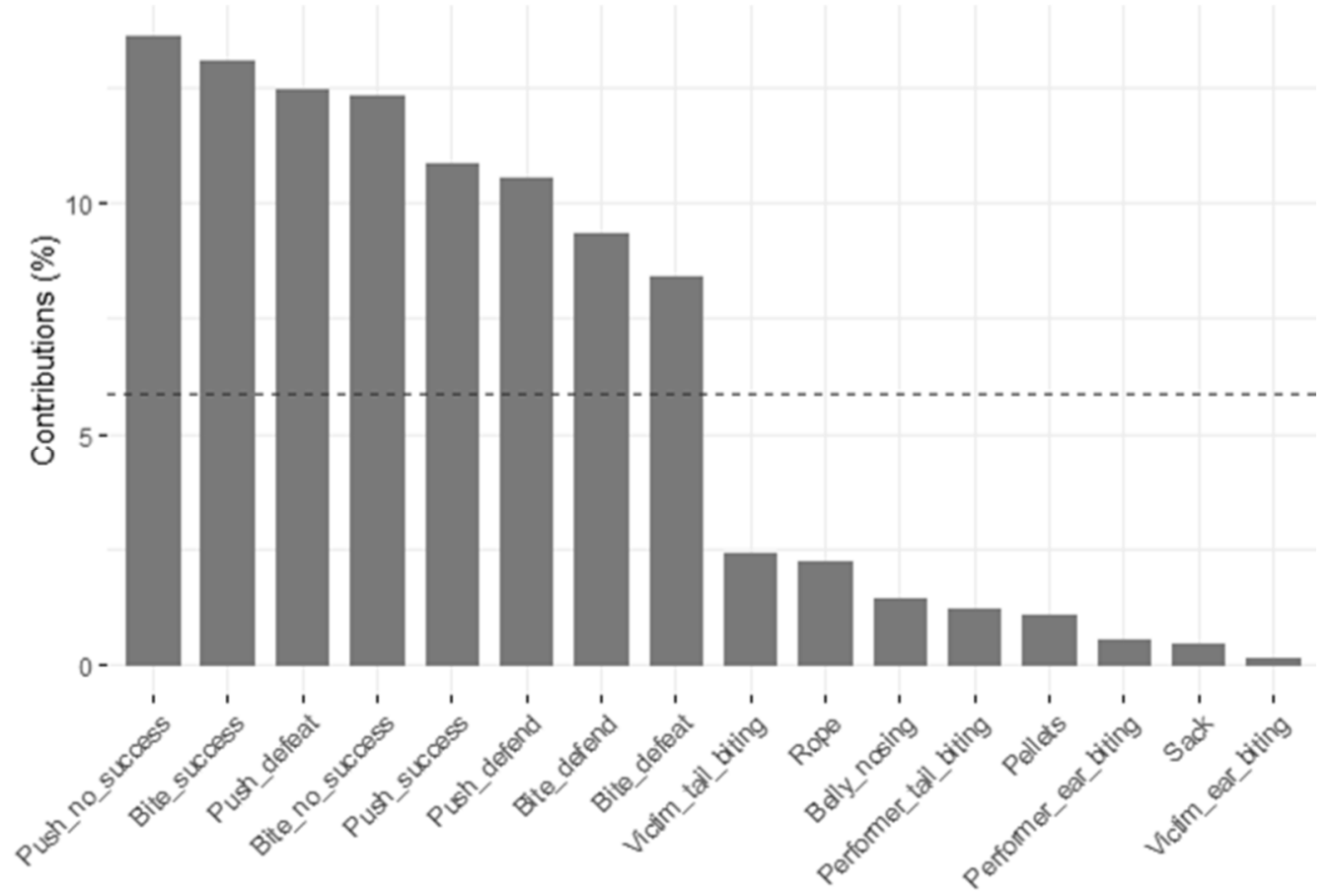

Figure 7. Contribution of variables to principal component factors 1 and 2 for cluster 2; dashed line $=$ expected value if contribution were uniform.

\section{Discussion}

Tail biting in pigs is a complex behavior due to the high number of influencing factors, its low frequency and unpredictability. In this study, we observed manipulative behaviors in weaner pigs, including performers and victims of tail biting, and analyzed correlations with agonistic suckling behaviors.

\subsection{Manipulation Behaviors}

The frequencies of observed ear biting and belly nosing behavior varied over the whole experiment and were also affected by repetition. As weaning entails separation of the sow, transport to a new housing environment and mixing with unfamiliar conspecifics, it goes along with new and maybe stressful impressions for the piglets [54].

Especially after regrouping of unfamiliar pigs, vigorous fights were observed between them to establish a social hierarchy. Those fights are primarily characterized by bites, and therefore lesions at the head and ears of the competitors, but can also involve the flank of the opponent [55]. In our experiment, the percentage of recorded body side and ear injuries was highest in the first week (until day 35 of life) and the last two weeks of rearing (day 63 of life until end of rearing). For the first week, we suggest that these injuries were caused by fights for hierarchical order. The percentage of injured body sides was also correlated with pigs' weight at week ten (day 70 of life), indicating that heavier pigs were hurt more frequently. This may be explained by decreasing available space per pig with proceeding growth of the animals [56], which may explain the high percentages of body side and ear injuries in the last two weeks of rearing.

We observed highest frequencies of tail biting around day 47-52 and day 65 of life. Comparing the pens, the highest average of tail biting was reached in pen 5 of the first repetition, possibly as a result of "obsessive tail biting" performed by one specific pig [7]. This individual stood out by performing a multiple of tail biting behaviors compared to its pen mates with the maximum mean of 29.25 tail biting behaviors per hour on day 65 of life and overall, only $2.6 \%$ tolerating reaction of its victims. By defining "tail biting", no distinction was made between tail-in-mouth and visible biting of the tail. Thus, the severity of the biting was deduced from the victim's reaction. It is of particular interest to note that 
both performing and receiving tail bites was observed in most pigs as also described by Zonderland et al. and Hoy et al. [10,11].

No significant correlation was found between the frequency of performed tail biting and any frequency of other manipulation behaviors. The frequency of received tail bites was positively correlated with manipulation of the jute sack. This result disagrees with results of several authors who found biters to spend more time manipulating enrichment or other parts of the bodies of their pen mates than victims [10,18,32]. Nevertheless, it must be taken into account that our finding was expected, since the jute sack was only offered in pens with severe tail lesions shown by the majority of the pigs.

Within the weekly scorings, a first significant increase of the proportion of tail lesions between two consecutive evaluations was found for all tail lesion parameters on day 63 of life in repetition 1 and day 56 of life in repetition 2 . In both repetitions the increase continued until the end of the rearing period. Thus, in our experiment, those lesions occurred at least a week later than described by several authors who observed a first increase of tail lesions two to three weeks after weaning (day 42 to 49 of life) [24-29]. The presence of tail lesions at the end of rearing was positively correlated with the frequency of received tail bites. However, the lesions were not diagnosed until ten days after the notable increase in the frequency of tail biting (increase of tail biting at day 52 vs. increase of tail lesions at day 63 of life in repetition 1; increase of tail biting at day 47 vs. increase of tail lesions at day 56 of life in repetition 2). This delay confirms previous findings of Zonderland et al. [30], even though it was also influenced by the interval of a week between lesion evaluations. Moreover, it underlines the importance of behavioral observations for early detection of tail biting and thereby successful prevention of severe tail lesions.

\subsection{Relationship between Suckling and Rearing Behaviors}

After transfer and regrouping to the rearing facilities, we found considerable differences between the rearing pens. We found moderate negative but significant correlations of performed tail biting behavior with dominance index and weaning weight, respectively, indicating that light subdominant pigs have performed more tail biting behavior than heavier piglets and therefore confirm findings of Zonderland et al., Beattie et al. and van de Weerd et al. $[10,16,17]$. The lower weaning weight might be caused by a nutrient deficient during lactation [12]. To compensate that deficit, smaller piglets might use tail biting to chase other piglets from the feeder or another resource with restricted access [9].

For a deeper insight into the relationship between suckling and rearing behavior, we performed a principal component analysis including each agonistic suckling behavior and manipulative rearing behavior separately. The highest contribution to principal components 1 and 2 related to all pigs were made by the agonistic suckling behaviors, independently of the success or intensity of the described interaction. However, the contributions of manipulation behaviors are clearly smaller than those of suckling behaviors to both principal components. This result suggests that the pigs might be primarily similar in their suckling behavior. This may be explained by the differences of the behavioral recording during both suckling and rearing. In the suckling period, the agonistic interactions were only recorded during the short durations of the suckling bouts, but not for the remaining time. Therefore, less variation in behavioral expressions is expected to occur compared to the traits recorded during rearing.

By separation of the pigs into two clusters, principal components of cluster 1 included main contributions of five different suckling behaviors, received tail biting and performed ear biting behavior, whereas principal components of cluster 2 were similar to principal components related to all pigs and thus primarily directed by the agonistic suckling behaviors. Since no significant correlation between those suckling and rearing behaviors was found and, furthermore, the pigs of a cluster did not fit properly in their cluster (silhouette width $=0.27$ ), we cannot derive a reliable connection between the analyzed agonistic suckling behaviors and manipulative rearing behaviors of pigs from this type of analysis. 


\section{Conclusions}

In our study, tail lesions were detected with a delay of about ten days, when an increase of tail biting behavior had already occurred. This underlines the importance of behavioral analysis for early detection and prevention of tail biting. The early identification of pigs predisposed for tail biting is hampered by the observed repeated shift in roles between biters and receivers, as also described by Zonderland et al. and Hoy et al. [10,11]. However, the observed moderate correlation between dominance index during suckling and performed tail biting during rearing warrants further research. To analyze further facets of tail biting behavior for the identification of pigs predisposed to tail biting, more aspects of pig personality as shown in early life should be considered.

Author Contributions: Conceptualization, F.K.W., A.L.v.A., T.S., and M.G. (Martina Gerken); methodology, F.K.W., M.G. (Mehmet Gültas), and M.G. (Martina Gerken); validation, F.K.W., M.G. (Mehmet Gültas), and M.G. (Martina Gerken); formal analysis, M.G. (Martina Gerken); investigation, F.K.W.; writing-original draft preparation, F.K.W.; writing—review and editing, A.L.v.A., M.G. (Mehmet Gültas), and M.G. (Martina Gerken); visualization, F.K.W.; supervision, M.G. (Martina Gerken); project administration, M.G. (Martina Gerken); funding acquisition, F.K.W. and M.G. (Martina Gerken). All authors have read and agreed to the published version of the manuscript.

Funding: This study was supported by the PhD program "Animal Welfare in Livestock Production Systems-transformation processes in intensive livestock farming" under the Lower Saxony Ministry of Research and Culture, Germany.

Institutional Review Board Statement: The study was conducted according to the guidelines of the Declaration of Helsinki, and approved by the Ethics Committee of the Agricultural Test Center VBZL Haus Duesse. No invasive interventions were performed on the animal. The study was conducted in accordance with the German Animal Protection Law and the Animal Welfare Livestock Farming Regulation.

Data Availability Statement: Not applicable.

Acknowledgments: The authors thank the employees of the Agricultural Test Center VBZL Haus Duesse of the Agricultural Chamber of North Rhine-Westphalia in Bad Sassendorf, Germany, for priceless help with housing the animals and scoring of pigs and pigtails. We thank Philip Kramer, Witzenhausen University, for video analyses. We also wish to thank Jürgen Dörl, University of Goettingen, for technical help and Sebastian Zeidler, University of Goettingen, for statistical advice.

Conflicts of Interest: On behalf of all authors, corresponding author states that there is no conflict of interest.

\section{References}

1. Fraser, D. Attraction to blood as a factor in tail-biting by pigs. Appl. Anim. Behav. Sci. 1987, 17, 61-68. [CrossRef]

2. Day, J.; Kyriazakis, I.; Lawrence, A. The effect of food deprivation on the expression of foraging and exploratory behaviour in the growing pig. Appl. Anim. Behav. Sci. 1995, 42, 193-206. [CrossRef]

3. Camerlink, I.; Bijma, P.; Kemp, B.; Bolhuis, J.E. Relationship between growth rate and oral manipulation, social nosing, and aggression in finishing pigs. Appl. Anim. Behav. Sci. 2012, 142, 11-17. [CrossRef]

4. European Food Safety Authority (EFSA). The Risks Associated with Tail Biting in Pigs and Possible Means to Reduce the Need for Tail Docking Considering the Different Housing and Husbandry Systems-Scientific Opinion of the Panel on Animal Health and Welfare. EFSA J. 2007, 5, 611. [CrossRef]

5. Buijs, S.; Muns, R. A Review of the Effects of Non-Straw Enrichment on Tail Biting in Pigs. Animals 2019, 9, 824. [CrossRef]

6. Kritas, S.K.; Morrison, R.B. Relationships between tail biting in pigs and disease lesions and condemnations at slaughter. Veter. Rec. 2007, 160, 149-152. [CrossRef]

7. Taylor, N.R.; Main, D.C.; Mendl, M.; Edwards, S.A. Tail-biting: A new perspective. Veter. J. 2010, 186, 137-147. [CrossRef] [PubMed]

8. Van Putten, G. An Investigation into Tail-Biting among Fattening Pigs. Br. Veter. J. 1969, 125, 511-517. [CrossRef]

9. D'Eath, R.B.; Arnott, G.; Turner, S.P.; Jensen, T.; Lahrmann, H.P.; Busch, M.E.; Niemi, J.K.; Lawrence, A.B.; Sandøe, P. Injurious tail biting in pigs: How can it be controlled in existing systems without tail docking? Animals 2014, 8, 1479-1497. [CrossRef] [PubMed]

10. Zonderland, J.J.; Schepers, F.; Bracke, M.B.M.; Hartog, L.A.D.; Kemp, B.; Spoolder, H.A.M. Characteristics of biter and victim piglets apparent before a tail-biting outbreak. Animals 2011, 5, 767-775. [CrossRef] [PubMed] 
11. Hoy, S.; Engel, D.; Jans-Wenstrup, I. Ethological investigations on the perpetrators and victims of tail biting in weaner pigs. Livest. Sci. 2020, 231, 103879. [CrossRef]

12. Prunier, A.; Averos, X.; Dimitrov, I.; Edwards, S.A.; Hillmann, E.; Holinger, M.; Ilieski, V.; Leming, R.; Tallet, C.; Turner, S.P.; et al. Review: Early life predisposing factors for biting in pigs. Animals 2020, 14, 570-587. [CrossRef]

13. Schrøder-Petersen, D.; Simonsen, H. Tail Biting in Pigs. Veter. J. 2001, 162, 196-210. [CrossRef]

14. Widowski, T. Causes and Prevention of Tail Biting in Growing Pigs: A Review of Recent Research. In Proceedings of the 2nd London Swine Conference, London, ON, Canada, 11-12 April 2002; p. 47.

15. Sonoda, L.T.; Fels, M.; Oczak, M.; Vranken, E.; Ismayilova, G.; Guarino, M.; Viazzi, S.; Bahr, C.; Berckmans, D.; Hartung, J. Tail biting in pigs-causes and management intervention strategies to reduce the behavioural disorder. A review. Berliner Münchener Tierärztliche Wochenschrift 2013, 126, 104-112.

16. Beattie, V.E.; Breuer, K.; O'Connell, N.E.; Sneddon, I.A.; Mercer, J.T.; Rance, K.A.; Sutcliffe, M.E.M.; Edwards, S.A. Factors identifying pigs predisposed to tail biting. Anim. Sci. 2005, 80, 307-312. [CrossRef]

17. Van De Weerd, H.A.; Docking, C.M.; Day, J.E.L.; Edwards, S.A. The development of harmful social behaviour in pigs with intact tails and different enrichment backgrounds in two housing systems. Anim. Sci. 2005, 80, 289-298. [CrossRef]

18. Ursinus, W.W.; Wijnen, H.J.; Bartels, A.C.; Dijvesteijn, N.; Van Reenen, C.G.; Bolhuis, J.E. Damaging biting behaviors in intensively kept rearing gilts: The effect of jute sacks and relations with production characteristics. J. Anim. Sci. 2014, 92, 5193-5202. [CrossRef] [PubMed]

19. Breuer, K.; Sutcliffe, M.; Mercer, J.; Rance, K.; O'Connell, N.; Sneddon, I.; Edwards, S. Heritability of clinical tail-biting and its relation to performance traits. Livest. Prod. Sci. 2005, 93, 87-94. [CrossRef]

20. Munsterhjelm, C.; Simola, O.; Keeling, L.; Valros, A.; Heinonen, M. Health parameters in tail biters and bitten pigs in a case-control study. Animals 2013, 7, 814-821. [CrossRef]

21. Van de Weerd, H.A.; Docking, C.M.; Day, J.E.; Avery, P.J.; Edwards, S.A. A systematic approach towards developing environmental enrichment for pigs. Appl. Anim. Behav. Sci. 2003, 84, 101-118. [CrossRef]

22. Bracke, M.B.; Zonderland, J.J.; Lenskens, P.; Schouten, W.G.; Vermeer, H.; Spoolder, H.A.; Hendriks, H.J.; Hopster, H. Formalised review of environmental enrichment for pigs in relation to political decision making. Appl. Anim. Behav. Sci. 2006, 98, 165-182. [CrossRef]

23. Studnitz, M.; Jensen, M.B.; Pedersen, L.J. Why Do Pigs Root and in What Will They Root? A Review on the Exploratory Behaviour of Pigs in Relation to Environmental Enrichment. Appl. Anim. Behav. Sci. 2007, 107, 183-197. [CrossRef]

24. Zonderland, J.J.; Wolthuis-Fillerup, M.; Van Reenen, C.G.; Bracke, M.B.; Kemp, B.; Hartog, L.A.D.; Spoolder, H.A. Prevention and treatment of tail biting in weaned piglets. Appl. Anim. Behav. Sci. 2008, 110, 269-281. [CrossRef]

25. Abriel, M.; Jais, C. Influence of Housing Conditions on the Appearance of Cannibalism in Weaning Piglets. Landtechnik 2013, 68, 389-393.

26. Abriel, M.; Jais, C.; Bernhardt, H. Influence of Pen Design and Space Allowance on Tail Biting in Weaning Piglets. Landtechnik 2014, 69, 308-314.

27. Veit, C.; Traulsen, I.; Hasler, M.; Tölle, K.-H.; Burfeind, O.; Beilage, E.G.; Krieter, J. Influence of raw material on the occurrence of tail-biting in undocked pigs. Livest. Sci. 2016, 191, 125-131. [CrossRef]

28. Jans-Wenstrup, I. Untersuchungen Zur Prävention der Caudophagie Bei Absetzferkeln Unter Besonderer Berücksichtigung Einer Pelletzulage. Ph.D. Thesis, Justus Liebig University, Gießen, Germany, 2018.

29. Chou, J.-Y.; O'Driscoll, K.; D’Eath, R.B.; Sandercock, D.A.; Camerlink, I. Multi-Step Tail Biting Outbreak Intervention Protocols for Pigs Housed on Slatted Floors. Animals 2019, 9, 582. [CrossRef]

30. Zonderland, J.J.; Kemp, B.; Bracke, M.B.M.; Hartog, L.A.D.; Spoolder, H.A.M. Individual piglets' contribution to the development of tail biting. Animals 2011, 5, 601-607. [CrossRef]

31. Camerlink, I.; Turner, S. The pig's nose and its role in dominance relationships and harmful behaviour. Appl. Anim. Behav. Sci. 2013, 145, 84-91. [CrossRef]

32. Brunberg, E.; Wallenbeck, A.; Keeling, L.J. Tail biting in fattening pigs: Associations between frequency of tail biting and other abnormal behaviours. Appl. Anim. Behav. Sci. 2011, 133, 18-25. [CrossRef]

33. Ursinus, W.W.; Van Reenen, C.G.; Kemp, B.; Bolhuis, J.E. Tail biting behaviour and tail damage in pigs and the relationship with general behaviour: Predicting the inevitable? Appl. Anim. Behav. Sci. 2014, 156, 22-36. [CrossRef]

34. Larsen, M.L.V.; Andersen, H.M.-L.; Pedersen, L.J. Changes in activity and object manipulation before tail damage in finisher pigs as an early detector of tail biting. Animals 2019, 13, 1037-1044. [CrossRef] [PubMed]

35. Schrøder-Petersen, D.L.; Heiskanen, T.; Ersbøll, A.K. Tail-in-mouth behaviour in slaughter pigs, in relation to internal factors such as: Age, size, gender, and motivational background. Acta Agric. Scand. Sect. A Anim. Sci. 2004, 54, 159-166. [CrossRef]

36. Paoli, M.; Lahrmann, H.; Jensen, T.; D'Eath, R. Behavioural differences between weaner pigs with intact and docked tails. Anim. Welf. 2016, 25, 287-296. [CrossRef]

37. Lahrmann, H.P.; Hansen, C.F.; D’Eath, R.; Busch, M.E.; Forkman, B. Tail posture predicts tail biting outbreaks at pen level in weaner pigs. Appl. Anim. Behav. Sci. 2018, 200, 29-35. [CrossRef]

38. Lahrmann, H.P.; Hansen, C.F.; D’Eath, R.B.; Busch, M.E.; Nielsen, J.P.; Forkman, B. Early intervention with enrichment can prevent tail biting outbreaks in weaner pigs. Livest. Sci. 2018, 214, 272-277. [CrossRef] 
39. Larsen, M.L.V.; Andersen, H.M.-L.; Pedersen, L.J. Tail posture as a detector of tail damage and an early detector of tail biting in finishing pigs. Appl. Anim. Behav. Sci. 2018, 209, 30-35. [CrossRef]

40. Wedin, M.; Baxter, E.M.; Jack, M.; Futro, A.; D’Eath, R.B. Early indicators of tail biting outbreaks in pigs. Appl. Anim. Behav. Sci. 2018, 208, 7-13. [CrossRef]

41. Wallgren, T.; Larsen, A.; Gunnarsson, S. Tail Posture as an Indicator of Tail Biting in Undocked Finishing Pigs. Animals 2019, 9, 18. [CrossRef]

42. Warns, F.K. Identification of Dominance in Suckling Piglets. Caudophagie bei Mastschweinen (Sus Scrofa) Während der Ferkelaufzucht: Früherkennung und Prävention durch Umweltanreicherung. Ph.D. Thesis, Georg August University, Goettingen, Germany, 2020; pp. 49-77.

43. Welfare Quality. Welfare Quality ${ }^{\circledR}$ Assessment Protocol for Pigs (Sows and Piglets, Growing and Finishing Pigs); Welfare Quality®Consortium: Lelystad, The Netherlands, 2009.

44. Friedrich Loeffler Institute (FLI). Deutscher Schweine Bonitur Schlüssel; FLI: Greifswald, Germany, 2017.

45. Bowen, D.W.; Brooks, R.J. Social organization of confined male collared lemmings (Dicrostonyx groenlandicus Traill). Anim. Behav. 1978, 26, 1126-1135. [CrossRef]

46. Craig, J.; Biswas, D.; Guhl, A. Agonistic behaviour influenced by strangeness, crowding and heredity in female domestic fowl (Gallus gallus). Anim. Behav. 1969, 17, 498-506. [CrossRef]

47. Hope, A.C.A. A Simplified Monte Carlo Significance Test Procedure. J. R. Stat. Soc. Ser. B. 1968, 30, 582-598. [CrossRef]

48. Chambers, J.M.; Freeny, A.E.; Heiberger, R.M. Analysis of Variance; Designed Experiments. Stat. Models S 2017, 5, 145-193. [CrossRef]

49. Yandell, B. Practical Data Analysis for Designed Experiments; Routledge: Abingdon, UK, 2017.

50. Best, D.J.; Roberts, D.E. Algorithm AS 89: The Upper Tail Probabilities of Spearman's Rho. J. R. Stat. Soc. Ser. C 1975, 24, 377-379. [CrossRef]

51. Wickham, H. Getting started with qplot. In ggplot2; Springer: Berlin/Heidelberg, Germany, 2009; pp. 9-26.

52. Lê, S.; Josse, J.; Husson, F. FactoMineR: An R Package for Multivariate Analysis. J. Stat. Softw. 2008, 25, 1-18. [CrossRef]

53. Kassambara, A.; Mundt, F. Factoextra: Extract and Visualize the Results of Multivariate Data Analyses. R Package Version 1.5. 2017. Available online: https:/ / cran.r-project.org/web/packages/factoextra/factoextra.pdf (accessed on 19 April 2021).

54. D'Eath, R.B. Socialising piglets before weaning improves social hierarchy formation when pigs are mixed post-weaning. Appl. Anim. Behav. Sci. 2005, 93, 199-211. [CrossRef]

55. Meese, G.; Ewbank, R. The establishment and nature of the dominance hierarchy in the domesticated pig. Anim. Behav. 1973, 21, 326-334. [CrossRef]

56. Ewbank, R.; Bryant, M. Aggressive behaviour amongst groups of domesticated pigs kept at various stocking rates. Anim. Behav. 1972, 20, 21-28. [CrossRef] 